\title{
LISTEN AND LEARN SESSIONS
}

\section{Accessibility Is in the Eye of the Beholder:}

\author{
Creating a More Accessible Library
}

Kenneth D. Litwak, Gateway Seminary

\begin{abstract}
According to the U.S. National Center for Education Statistics, $19 \%$ of undergraduates report having a disability. Many of these students access our libraries in person or online. While theological and academic librarians recognize the need to accommodate people with disabilities, librarians without disabilities might not be aware of ways in which their library places obstacles in the way of those with disabilities, such as computers that cannot have the font color or size changed or steps but no ramps for wheelchairs. This essay describes issues for patrons with disabilities in accessing library services and resources. There are several ways to improve the accessibility of libraries. This essay highlights challenges for those with visual, auditory, and mobility disabilities and describes ways to address these challenges and thus give those with disabilities a more equitable, inclusive library experience.
\end{abstract}

\section{THE BASIC PROBLEM}

Many of the students who would benefit from using the library at their college, university, or seminary have one or more disabilities. According to the National Center for Education Statistics, 19\% of undergraduates report having a disability. Twelve percent of graduate students have reported having a disability (NCES, 2019). Librar- 
ians seek to serve all patrons. However, some of those patrons need accommodations in order to use a library's services and resources. Many patrons at academic and theological higher educational institutions have disabilities. Some are apparent, such as visual impairment or mobility problems as evidenced by a patron's wheelchair. There are other sorts of disability, many "invisible," but this essay deals with apparent disabilities, those that can be seen or readily perceived. There are several challenges for patrons with disabilities. There are two approaches to describing such individuals: a disabled person or a person with a disability. This essay uses and recommends the latter, since "a disabled person" defines someone by her disability, which is not the most important feature of the person. We do not want to treat people as a problem to solve but a person whom we can help.

Those without disabilities might not "see" the (unintentional) challenges the library presents. For example, if the instructions on the copier are in 10-point Times New Roman, some patrons will have difficulty reading them. The same holds true for signage with relatively small fonts, including directional signs and call number ranges on the ends of shelving. Some patrons may have difficulty in moving compact shelving. Those without any physical or sensory challenges might not see these things as problems, but for patrons with specific disabilities, these are major obstacles.

A second problem is that patrons with disabilities may be uncomfortable disclosing their disability to a librarian who does not have one. Disclosure can be difficult enough. If the library staff does not know that an accommodation or service is needed, it will not offer the accommodation. Or, a service or accommodation does exist, such as a library computer with a screen reader, but there is nothing indicating the existence of this computer for those with visual impairments or blindness. Here are some specific types of problems by the type of the disability.

\section{VISUAL IMPAIRMENT AND BLINDNESS}

Many people have visual impairments, such as having significant near-sightedness that cannot be readily corrected, or blindness. Below are some challenges for individuals with such conditions. 


\section{Difficulty Accessing or Configuring Computers}

If the monitor is too far away from the front of the piece of furniture it is on, or the settings, such as font and mouse pointer size, are too small and not adjustable, a library computer can be inaccessible to some patrons with a visual impairment. I have a significant visual impairment. While attending a conference at a Christian university, I attempted to use a library computer to check email. I did not succeed. First, the monitor was at least eighteen inches back from the edge of the desk it was on. It was bolted in place for security. Due to this, I had to stand up and lean far over the desk to read the screen at all. Second, the font size for text appeared small, too small for me to read, even with high-powered reading glasses. The mouse pointer was also quite small. The computer had been configured to prevent any changes at all. I could not increase the font size or change the mouse pointer. Therefore, I left the library after being unable to complete my task. The configuration of the computer may have been fine for someone with "average" eyesight, but it was not accessible for someone like me.

\section{0/20 Vision Is Assumed}

This relates to the issues above about being able to configure a computer screen, but it also relates to other areas. Handouts, equipment instructions, and signage are a challenge for someone with a visual impairment, and these items are often not machine-readable, while most patrons have no trouble with instructions or handouts that use a 10-pt. Times New Roman font. Even if one had the equipment to read a document, if it is not OCR-compliant, various tools are not going to be able to read it. One could also ask: Why are call number ranges put at the top of shelving? That is another issue for those with visual impairments. It can seem like many resources in the library are actually an eyesight test. Hanging signs from the ceiling can be difficult to read as well. It is fine to have a hanging sign, but the font should be larger than the "average" patron can read.

\section{No Screen Magnifiers or Readers}

There are several tools, like NVDA, that can be used to make what is on the screen larger, and some can read what is on the screen, such as JAWS. However, many academic and theological libraries do not have this sort of software, because no one has thought about it being necessary for some patrons. These tools can greatly help patrons 
with vision problems but they are often not available. When they are, it is not always obvious which computers have this software. Even when there is a computer with a screen reader, blind users without essential knowledge may have difficulty using the software, and it is often the case that librarians are not sufficiently trained in assistive technology to help such patrons. Other webpage issues are related to navigation. The ubiquitous three dots that represent menus might not have alt-text. A screen reader like JAWS won't know what to do with a column of dots.

\section{Web Pages Are Inaccessible}

Many library websites have images. Those images often have no alt-text and therefore a blind user only gets to know that an image is there. For her, that image is meaningless. Mulliken states that for those using screen readers, "Pages with a very large amount of content, such as many library and vendor pages, can be particularly overwhelming, especially if not coded with logically placed headings and other features to allow a sensible navigational path for blind users” (Mulliken, 2017, 117).

\section{PDF Files Are Not Machine-Readable}

Libraries create PDF files regularly. At Gateway Seminary, like many schools, books and journal articles are scanned or copied and the resulting PDF files are sent to patrons. What if a patron needs the PDF file to be machine-readable for his software? PDF files are an accessibility challenge.

\section{Lack of Vendor Support for the Print-Disabled}

While not everyone who is print-disabled has a visual impairment, the lack of vendor support for those who are print-disabled is an issue. If someone cannot use printed materials, a library without software to read the text to the patron will not meet the patron's needs. One area this affects is e-books. If a patron can see the book on the screen but cannot readily read it or scroll or move the text around by mouse or keyboard, it will be difficult for this person to use this resource. While some vendors, such as JSTOR, provide text-to-speech tools, other vendors, such as ProQuest Ebook Central, provide no such tools. This is an issue for both those who are visually impaired and those who are print-disabled for other reasons. 


\section{Stairs Not Clearly Marked}

If you have ever tripped on stairs because you missed one or did not step on one adequately, it is not hard to understand the potential injury that a lack of good depth perception could cause a patron on stairs. The problem is that if a person cannot tell how far down the next step is, she might miss the step or fall on to the next step too hard.

\section{AUDITORY DIFFICULTIES}

Those with hearing problems also encounter difficulties in the library.

\section{Cannot Hear Video Tutorials}

That tutorial, which took many hours to record and edit, will be of limited value to someone who cannot hear the narration. Typically, video tutorials on library websites do not offer closed captioning. They tend to lack transcripts of tutorials as well. With no written script or captioning, video tutorials are generally unhelpful to those with limited hearing.

\section{Instruction with No Written Content and No Sign-Language Interpreter}

The same problem presented by video tutorials that have no captions or transcripts is present for live information literacy instruction. The content may be excellent but without some way to know what was said, it will be difficult for someone who is hearing-impaired or deaf to gain much from the ILI session. The patron with the auditory disability may know sign language. If there is no one present to offer the instruction in sign language, this will be another lost opportunity to offer instruction to all patrons. If written material or sign language is available, a patron might not know about it or think to ask about it. Instead, he will simply skip the instruction, no matter how much it is needed.

\section{Lack of Braille Labels}

Another challenge for blind patrons is a lack of Braille signs or identifiers, such as for elevator buttons. This is unique to someone who is blind or has severely limited eyesight. However, we do not want to leave anyone out because their disability is not common among our patrons. 


\section{MOBILITY DISABILITIES}

Other challenges are faced by those with mobility issues. This could include the use of a wheelchair or walker, inability to stand on a stool to get a book of the high, top shelf, or other obstacles.

\section{Lack of Ramps}

Those using wheelchairs, walkers, or crutches, or with some sort of brace on a leg, will likely have difficulty going up or down stairs. This is different from the lack of clearly marked stair edges. It is the fact that wheelchairs cannot go up or down stairs. Some libraries have elevators that can reach every floor. Others have ramps that those with mobility issues can use to go up or down. However, some libraries have places where there is no way to go up or down except by using stairs.

\section{Stacks Are Too Tall to Reach Top Shelves from a Wheelchair}

The stacks are often quite tall. Someone in a wheelchair or with other mobility issues might not be able to reach the top shelf/shelves. I was impressed when I went to the James L. Stamps Theological Library at Azusa Pacific University. Most of the reference books were in short shelving units with only two shelves. One could put a laptop on top of the shelving units, find a reference book in the catalog, and easily reach the book. Some libraries might not have space for such shelving, but it is a great accommodation for many people with mobility disabilities or visual impairment.

\section{Compact Shelving May Be Difficult to Move}

Many libraries use compact shelving to save space. It seems like a good idea, as long as one is able to operate it. When Gateway Seminary was in northern California as Golden Gate Baptist Theological Seminary, many books and almost all the periodicals were in compact shelving. The shelving units used mechanical means to move the shelves. One touched a button on the desired shelving unit, and all the shelving units in the way of it moving were moved by the system. Gateway Seminary's Ontario Library does not have this sort of compact shelving. It is necessary to use handles to move shelving. This is not too difficult with one arm to move one shelf. Moving multiple shelves is another matter. It can take significant effort to move multiple shelving units. If one cannot use their arms or hands 
to accomplish this, the patron will be unable to access any books on the shelves. The only way to solve this would be for the patron to go to the library circulation desk, which is one floor up, and ask for help. Compact shelving is a fine idea. It does conserve space. Unfortunately, some patrons will not be able to use it. While some academic libraries have a "call" system to get help in retrieving books from a high shelf or other locations that are hard for some to access, not all libraries do. This may be particularly an issue for small seminary libraries that have a small staff.

\section{LACK OF INFORMATION ON ACCESSIBILITY AND ACCOMMODATIONS}

Patrons with disabilities want to know the sorts of accommodations available at the physical library. Amelia Brunskill presented survey results for students with disabilities who said that without a page on a library website that lists accommodations and other ways the library is accessible for all, these students would simply not go to the library. As noted near the beginning of this essay, many patrons with disabilities do not wish to disclose their disabilities to a librarian who does not have any disabilities. Instead, they want to find out information on accessibility on the library website. Brunskill interviewed twelve students with disabilities about "their perspectives on navigation, search terms, organization, desired content, and the overall look and feel for such a webpage" (Brunskill, 2020, 768). Many libraries do not have this information on a webpage, even if they have accommodations for students. This apparently communicates to students that a given library does not provide accommodations.

\section{MAKING LIBRARIES MORE ACCESSIBLE}

In order to deal with these issues and more, librarians will need to take an active part in making libraries more accessible. First, however, we need to answer the question: Why make accommodations in the library, both physically and digitally?

\section{Serve Patrons}

Our profession and all the individual librarians I have interacted with seek to serve patrons. To borrow from an old sci-fi television series, "It's what I do." While there has been significant emphasis 
rightly placed on serving racial and ethnic minorities, people with disabilities also deserve equity and inclusion in library services and use of resources.

\section{Worldview}

Another answer to the "why" question comes from one's worldview. Should everyone get equal treatment? I work at an Evangelical seminary and loving the neighbor by serving them is a theological imperative. Many other worldviews would also consider helping others part of living out the worldview, such as Islam and Judaism. It does not require a religious perspective to be concerned about those with disabilities. Many with humanist worldviews take care of people on the margins.

\section{Legal Issues}

Within the U.S. and Canada, libraries are expected to assist patrons with accommodations. In the U.S., most academic libraries must comply with Section 508 and the Americans with Disabilities Act. In California, there are even more laws regarding accessibility, as there are in many other states. Many academic institutions have had to pay out settlements from lawsuits over violating these laws. Therefore, it makes good fiscal sense, among other reasons, to seek to comply with them.

\section{GENERAL APPROACH}

Before describing approaches for addressing accessibility issues, there are general principles to guide a library.

\section{Make Accessibility Part of Planning}

Most of the accessibility issues already exist in libraries. When new technology or new instructional approaches or new library spaces are being considered, it is valuable to avoid problems by planning for accessibility and making it a high priority in the decision-making process.

\section{Audit the Environment}

To determine what needs to be addressed, a library can do an audit of its own environment, practices, equipment, and other tools. There 
are checklists to help a library perform an audit. There are also tools for auditing the library's website. Patrons with disabilities should be included in the discussion as they know better than anyone else what they need and wish for. When accommodations are made, asking (or bribing) these patrons to test the accommodations is important. I worked as a computer programmer for many years and taught software testing. One important element is that testing for the expected use of the software is not sufficient. It needs to be tested by experienced people who will put the software through its paces and go far beyond the "normal" use of a program. It's also important to devise tests for unexpected actions by those who do not understand what they are supposed to do. Likewise, having librarians without impairments test accommodations, like using a screen reader to perform a search or go up a ramp to the circulation desk is not sufficient. The "expert" users who really need the accommodations need to participate in evaluating them. The feedback from those with disabilities can be invaluable in determining if a library's accommodations make any meaningful difference.

Many academic libraries have already taken steps to make the library physically and digitally more accessible. This essay is not a blanket condemnation of all academic libraries. At other libraries, there are many opportunities to provide accommodations. Below are suggestions for making a library accessible that mirror the problem areas described above. Some of these items will be "free" in that no money or little effort is required to make the accommodation. Other items may cost more. Libraries at schools with an office for disabled students may be able to get help from there to defray costs.

\section{MAKING COMPUTERS MORE ACCESSIBLE}

An accessible workstation will provide easy access for wheelchair users. It may be fine to put computers and chairs relatively close together, but there needs to be a workstation that has space for a patron in a wheelchair to access it. It must be possible to configure a workstation completely, making the font bigger, making the contrast higher or lower, and changing the resolution of the monitor. My choice on MS Windows 10 is a medium resolution, $1680 \times 1050$. Selecting the lowest possible resolution, depending upon the monitor size, can result in large, black, vertical "bars" on both sides of the screen. This is wasted screen real estate. I am not a Mac user, but I 
have seen a user make the content of the screen denser. It is a retina setting. When I am trying to make what is on the screen larger, the last thing I want to do is waste two to four inches on the sides.

In Windows 10, the size of text displayed by the system in some contexts can be altered, such as making it $125 \%$ of the default. This may make text easier for some to see, but it does have a downside in that it can result in text being cut off. However, I have not had any problems with $125 \%$.

Colors are also important. Be careful using colors on library pages that might not be visible to someone who is color-blind. Having too many bright colors can be an issue for some. It is a matter of taste whether a user with visual impairment wants black text on a white background or white text on a black background. Being able to change the text and background color can be very helpful.

The mouse pointer can be an issue too. Having a significant visual impairment, I cannot count all the time I have wasted trying to find a tiny mouse pointer. It would help if the pointer was a more obvious color, like yellow or neon orange. It can also be helpful, again on Windows, to set the mouse pointer to be dark on light backgrounds and light on dark backgrounds.

Enabling patrons to change all these settings is not a problem. A Windows computer can be put into “deep freeze.” A patron can log in, make all the changes that she wants, and when she logs out or times out, the computer display will go back to the default chosen for all the library computers. I first encountered this at Azusa Pacific University, where I was an adjunct reference librarian. I could change a display to suit my needs but upon logging off, the workstation returned to its default. There was no need to worry if a patron modified the configuration significantly. The computers were very accessible.

Screen magnifiers and readers are also important tools for those with visual impairments or blindness. There are multiple ways to magnify the screen. It is possible to put a cover over the front of the monitor that can enlarge what is on the screen. Better is using screen magnifier software. Again, speaking within an MS Windows 10 context, there is a Magnifier utility. In previous versions of Windows, the magnifier provided a horizontal "slice" of the screen where the mouse pointer was. This was difficult to use. Now, the magnifier can be set to magnify the entire screen. The magnification is easily adjustable. There are other screen magnifier programs, such as NVDA and the far more expensive ZoomText. It is also important to 
provide screen readers for those with visual impairments or blindness. JAWS is a common screen reader. It can identify buttons, dropdown lists, etc. that have alt-text. It can also read text on the screen. ZoomText, which I have used for years, can also read the text on the screen, though it does not "read" buttons or other controls. Such tools are a great relief for those with visual impairments. It reduces eye strain and having to lean over the keyboard to read the screen, which can literally be a pain in the neck. The options vary widely in price. For those who use mobile devices, there are phone apps that can read the screen.

It is essential that a patron can interact with the computer by the keyboard or the mouse. There is no guarantee that a patron with disabilities will be able to use the mouse, the common tool for selecting buttons and other controls. In addition, a touch screen can be helpful, not only to those with mobility disabilities but those with a common condition from computer use, Carpal Tunnel Syndrome. This can make moving the mouse very painful. Being able to click a button or select something on the screen by touch, or using the touch on-screen keyboard, can be great assistance for some patrons.

Using text-to-speech tools in the library makes it mandatory to have headphones available to avoid disturbing other patrons. For some patrons, it would be very helpful to have speech-to-text tools for input purposes, such as Nuance's Dragon Naturally Speaking, though there may be licensing issues with it on a publicly available computer.

Finally, when it comes to assistive technology, it is not necessarily true that if we build it, patrons with disabilities will use it. It is essential that such workstations be well advertised. Patrons need to be able to find out easily that a computer with one or more of these accommodations exists. It is also important to make sure that all the librarians know about which assistive technology is available and how to use it. If a patron comes to the library who would benefit from a screen reader but does not know how to use one, it will be up to the librarians to teach him. 


\section{ADDRESSING ISSUES FOR THOSE WITH VISUAL IMPAIRMENT OR BLINDNESS}

There are several steps that a library can take to help patrons with visual impairments or blindness. Here are some important ways to accomplish this goal.

\section{Accessible Signage}

All signage needs to be in large print. This applies to everything from an accessible workstation with a screen reader to a photocopier or printer. Good options can be 28, 32,36, and other font sizes. It does not matter if the typical patron can read 10-pt. Times New Roman easily; those with visual impairments often find small fonts like that difficult if not impossible to read. Our goal is to remove all the obstacles to patrons accessing our services and resources, which means that librarians are not the primary audience for signage. If a student has to look really close to instructions to use a scanner, and needs to provide her own technology to magnify, we have, I would assert, failed that student. Patrons with disabilities should not need to bring any special tools to the library that those without disabilities do not need to bring. I have brought such tools to academic and theological libraries, and each experience reminds me that the environment and resources were designed for an exclusive group of patrons to which I do not belong. We do not want any patron to feel that way.

We could debate all day about which font is the best. When it comes to making accommodations, "best" is a very relative term. In addition to size, most users with visual impairments find the serifs difficult to read. I find it harder to distinguish characters in Times New Roman than in Arial. In creating LibGuides, I use Verdana 14 pt. This is an exception to the idea of not using a serif font. However, Verdana is a very large font. I do not want to have to squint at a sign or web page. There is no need for that when larger fonts and fonts with large sizes are readily available. Also, using a sans-serif font is no guarantee of being readable by all. Calibri is a sans-serif font but the characters are much smaller than, say, Arial.

Assume that the library has made sure its signage and instructions are in a large, easily readable font. That does not mean that someone who is significantly nearsighted can read it when the sign hangs from the ceiling and is ten feet above the floor. Certainly, signage should be visible to all, but it can do that and be at eye level. 
Deciding what eye level would mean at a given library cannot be specified in this article but it is most definitely not at the eye level of most NBA stars.

Instructions and handouts face the same need for easily readable fonts. These may be eye-level but hard to read. Be careful if something uses color that there is not a background color that makes text hard to read. It is not hard to find such things. I have even seen this problem in a commentary series that provides notes in the side margin in orange on a white background. That is not an accessible approach. Likewise, all instructions should be in large print and OCR-readable. PDF files also need to be accessible. This is a difficult issue. In my library, we can achieve that by telling the book scanner to produce black-and-white PDF files only. That usually generates machine-readable documents. The screen reader will not help a patron if a PDF file is essentially an image.

Stairs need to have the edges marked. Anyone who lacks full use of both eyes will have difficulty with proper depth perception. For myself, I can see the edges of stairs and see how tall a stair is when I am facing it and walking up. When walking down, I cannot do that. This is one of the simple, nearly free steps a library can take. Whether a library uses black, high-viz orange or black and yellow stripped tape, many patrons may benefit by a library marking the edges of steps.

\section{ADDRESSING ISSUES FOR AUDITORY DISABILITIES}

There are two main areas of concern for those with auditory disabilities.

\section{Video Tutorials}

As described above, having difficulty hearing or being deaf can make the best video tutorial nearly useless. The patron needs to know what the video narrator is saying. This can be addressed in two ways, and doing both is helpful. All video tutorials need to have closed captioning. This has some complexity. In Camtasia, there are two choices. A video can have optional closed captioning if additional files are uploaded with the video file itself. This may work on a library's web server but it will be a problem to upload additional files on websites such as Vimeo and YouTube. The second choice it to have Camtasia burn in the captioning. This makes the captions show up for 
everyone. This can be good, since multiple paths for input can often improve retention. Captions can help if a person can hear all that is said but not all the words are enunciated adequately. Camtasia can take recorded audio and automatically turn it into captions but the results I have had are rather poor. One solution for this is to create audio transcripts. There needs to be an audio transcript of the tutorial. The transcript can be used to build captions for the video. The transcript provides a description of the steps to perform whatever the tutorial was about. This can be useful to both those who could not hear the video and anyone else who wants instructions for tasks like searching the catalog or requesting ILL materials. For live video presentations, there are services that provide instant captioning. Atla used that for the 2021 annual conference. Companies such as Verbit provide such services.

\section{Live Instruction}

When offering instruction, such as information literacy instruction, it is important to include all potential patrons. Live instruction faces some of the same issues as accommodating those with visual impairment or auditory disabilities. It is possible with the right software to display instant captions. That will help those who cannot hear the presenter. For the session a librarian does over and over, an audio transcript would be helpful. The patron who cannot hear can use the transcript to know what is being said. That patron, along with everyone else, will have the contents of the instructions regardless of whether the audience takes notes or not. Another option would be for the library to provide someone who can use sign language to communicate with those who have auditory challenges.

\section{ADDRESSING ISSUES RELATED TO MOBILITY DISABILITIES}

For those who use wheelchairs, walkers, or other aids for walking, providing ramps to use instead of stairs is vital. Otherwise, many library resources and services may be inaccessible to these patrons. As our goal is to provide service and resources to all, practicing equity and inclusion, providing ramps is an obvious means of providing accommodations for such patrons.

For these and other patrons with mobility issues or other factors, the stacks need to be made more accessible. The call numbers should be at eye level like other signage. Short shelving is also important. 
This enables all patrons, including those in wheelchairs or of moderate height, to access resources off the shelf easily. This is important because, as noted above, many with disabilities do not want to disclose them or ask for "special" help. They want to be able access resources on their own. This also implies that compact shelving must be addressed. This might be a rather expensive recommendation, but installing compact shelving that uses push buttons for the shelving units to be moved mechanically, without having to turn a crank, is important.

\section{BUILDING ACCESSIBLE WEBSITES}

Since the library website is the key way that patrons interact with the library's services and resources, it must be accessible. There are tools to audit and describe how accessible a library's website is. For example, Axe from Deque or Wave are tools for testing a website's accessibility. The Digital Library Federation, DLF, also provides information and resources on auditing a library's website (DLF, 2021).

There is a group within the W3C for digital accessibility that has produced the widely accepted standard WCAG. It is currently at the 2.1 level, but by the time this is published, it will likely be at 2.2. There are varying levels of compliance. There is basic compliance that most websites can meet. On the other hand, implementing everything in WCAG 2.2 will be very challenging. WCAG addresses colors, fonts, controls, text, navigation, and more. Academic libraries should adopt WCAG 2.x and aim to be in compliance.

As suggested earlier, images, drawings, and controls such as buttons and drop-down lists need alt-text for screen readers to understand them. This should also apply to instruction. I have helped a blind student at our seminary who took a course in which the professor used lots of maps and images. These were unhelpful to her, as one could guess. Liaison librarians might reach out to faculty and raise this subject.

Some web pages are loaded with content. There are lots of buttons, sliders, and menus. There are also lots of images. These might make for cool-looking websites but they are not very accessible. As already noted, many of these cannot be processed by a screen reader, and having a lot of content can overwhelm a patron who has to use a screen reader to learn what is on the page. 
If the library has taken steps to provide accommodations, it needs to make these known on an accessibility information page on its website. As noted in Brunskill's study, many students with disabilities will not go to the library that does not provide such a page. It would be unfortunate if a library created accommodations only to have the patrons for whom the accommodations were done stay home, because they were unaware that someone was available to get books off of high shelves or that a computer had a screen reader and was configurable by the user.

\section{CONCLUSION}

Many obstacles stand in the way of people with visual, auditory, and mobility disabilities. There are also many steps academic and theological libraries can take to ameliorate these obstacles and make both their environment and website more accessible. Providing accommodations is the right thing to do because we want to serve patrons and avoid lawsuits. Libraries can audit their environment and their website. They can make signage easier to read, and they can make instructions, handouts, and PDF files more accessible by making them OCR-accessible. They can provide captioning for all video tutorials and audio transcripts for videos and live instruction. They can make sure that the library's website is in at least basic compliance with WCAG 2.1 and that the site has an accessibility information page. While free items that can be implemented are low-hanging fruit to work on first, the website is probably the most important, as it is the main way that patrons interact with libraries. Some libraries have made great strides in making themselves more accessible. Hopefully, other libraries will "go and do likewise."

\section{REFERENCE LIST}

Brunskill, Amelia. 2020. “'Without That Detail, I'm Not Coming”: The Perspectives of Students with Disabilities on Accessibility Information Provided on Academic Library Websites.” College \& Research Libraries 81 (5): 768-88. https://doi.org/10.5860/ crl.81.5.768. 
Digital Library Federation. 2021. "Accessibility Auditing Resources Accessibility Auditing Resources.” DLF Wiki. https://wiki.diglib. org/Accessibility_Auditing_Resources.

Mulliken, Adina. 2019. “Eighteen Blind Library Users’ Experiences with Library Websites and Search Tools in U.S. Academic Libraries: A Qualitative Study." College \& Research Libraries 80 (2): 152-68. https://search.ebscohost.com/login.aspx?direct=true\&db $=$ eric $\& A N=E J 1207603 \&$ site $=$ eds-live.

U.S. Department of Education, National Center for Education Statistics. (2021). Digest of Education Statistics, 2019 (2021-009), https:// nces.ed.gov/programs/digest/d19/ch_3.asp.

\section{NOTES}

1 Part of the problem is that ableism, the implicit belief that everyone is physically normal and those who are not cannot succeed, has a "choke hold" in higher education. It affects decisions that may affect an entire campus. 